\title{
BUILDING CHANGE DETECTION THROUGH COMPARISON OF A LIDAR SCAN WITH A BUILDING INFORMATION MODEL
}

\author{
H. Tran ${ }^{\text {a, } * \text { and K. Khoshelham }}{ }^{\mathrm{a}}$ \\ ${ }^{a}$ Department of Infrastructure Engineering, University of Melbourne, Parkville 3010, Australia - tran5@ student.unimelb.edu.au, \\ k.khoshelham@unimelb.edu.au
}

KEY WORDS: Indoor modelling, Point cloud, Automation, Change detection, Building Information Model (BIM), Temporal analysis.

\begin{abstract}
:
Building Information Models (BIMs) are of paramount importance in lifecycle management of buildings as they enable collaboration among various stakeholders at different phases of a construction project, from planning to maintenance and operation. However, there is usually inconsistency between the as-is condition of the building and its existing BIM, because BIMs are generally not updated to reflect changes in the environment. Monitoring the changes during a building's lifecycle and keeping the BIM up-to-date is useful for a variety of applications. Yet this process often involves manual surveying inspections, which are very time-consuming, error-prone, and laborious. In this paper, we present an automated approach for building change detection through a comparison between the BIM and a point cloud of the building indoor environment. The approach is based on point classification and surface coverage to identify discrepancies between the BIM and the point cloud. Experiments on a synthetic dataset and an ISPRS Benchmark dataset show the potential of the proposed approach not only for change detection and identifying discrepancies, but also for locating the removed and new structures of the building in comparison with the BIM. The results are useful for updating the BIM to represent the as-is condition of the building and for temporal analysis of changes during a building's lifecycle.
\end{abstract}

\section{INTRODUCTION}

Building Information Modelling is recognized as a key in digitalization of construction management. An as-is BIM of a building potentially increases collaboration, transparency and efficiency of information management as well as improvement in the decision-making process during the whole lifetime of the building, from planning to maintenance and operation (Hossain et al., 2018). However, it is common knowledge that an existing BIM may not be up-to-date and is not always consistent with the real environment.

BIM is now mostly available for new buildings at the design stage (EU BIM Task Group, 2017). However, during a building's lifetime, there are changes in the building structures introduced during the construction and the operation periods. While the construction phase often comprises various modifications of the design such as room dimensions, location and thickness of walls, and size of doors and windows (Choudhry et al., 2017), the maintenance and operation period involves renovation of building structures for different uses. In practice, the changes in one building part (e.g., interior structures) are likely to lead to modifications in other parts of a building (e.g., building façades and utilities). Hence, there is a need to manage and monitor the changes of a building during its lifecycle. Yet these discrepancies are often not well documented and continuously updated in the existing BIM. Therefore, there is a gap of information management across different periods of a building lifecycle. Additionally, monitoring the changes often involves manual surveying inspections, which are tedious, time-consuming, and error-prone (Wang et al., 2015). Consequently, the gap and inconsistencies between the existing BIM and the real building usually lead to errors and late deliveries and therefore increase the cost of the construction project.
Spatial data acquisition techniques, i.e. photogrammetry and laser scanning, enable effective capture of building interiors. Photogrammetric sensors capture and merge still images to generate 3D representations of the interior of buildings. The photogrammetric technique is generally efficient in time and cost. However, this technique suffers from shadows, changes of illumination conditions, and the presence of poorly textured surfaces (Khoshelham, 2018; Becker et al., 2018), which are common features of indoor environments and can cause failures in generating their 3D representation. The laser scanning technique is commonly more expensive than the photogrammetric solution. However, it allows direct generation of a point cloud as a 3D as-is representation of a building interior (Khoshelham, 2018). Laser scanning can produce highly accurate data (millimeters to a few centimeters) and is the preferable technique for capturing building interiors.

In the literature, Nikoohemat et al. (2018) proposed a method for change detection of a building interior by comparing the point clouds captured at different periods. However, a point cloud of a building is not always available, especially at the design stage. Meanwhile, the changes at the construction phase are significant, and monitoring the changes is necessary in order to eliminate errors and delay in the whole project. Khoshelham et al. (2018) introduced a method for comparison between two BIMs of an interior environment. This method enables identification of the missing and additional elements of the BIMs as well as the position discrepancies between them (Tran et al., 2019). However, despite intensive research on 3D indoor modelling (Khoshelham and Díaz, 2014; Díaz et al., 2015; Mura et al., 2016; Previtali et al., 2018, Tran et al., 2017, 2018), the generation of an as-is BIM for a complex interior building still requires a considerable effort and is known to be a time-consuming process.

In this paper, we propose a method for identification of discrepancies between an indoor environment and its existing

\footnotetext{
* Corresponding author
} 
BIM based on a comparison between the BIM and a point cloud of the environment. The method facilitates not only discrepancy detection, but also locating the missing and redundant elements of the BIM with respect to the as-is condition of the indoor environment. The main contributions of this work are as follows:

- An automatic method for comparison between a BIM and a point cloud of an indoor environment, which enables identification of the differences between them and facilitates change detection of an indoor environment;

- A method for effectively locating redundant and missing elements of a BIM in comparison with the as-is condition of the indoor environment, which is useful for updating the BIM and can be applicable to temporal analysis of buildings.

\section{METHOD FOR COMPARISON OF A BIM AND A POINT CLOUD OF A BUILDING INTERIOR}

We propose a method for comparison between a BIM $M$ and a point cloud $P$ of a building interior through identification of whether each point in the point cloud represents an element in the BIM, and likewise, whether each surface of the BIM is covered by points in the point cloud.

To facilitate the comparison, the BIM $M$ and the point cloud $P$ are first registered into a common coordinate system. The registration can be done automatically with available software (CloudCompare Development Team, 2019) by picking at least three corresponding points between them. Intuitively, the points of the point cloud $P$ representing a surface in the BIM $M$ are likely to be close to the surface. Likewise, a surface of the model $M$ which is existing in the building interior is mostly covered by the points. Therefore, to compare a BIM and a point cloud, we propose a method consisting of two main steps:

Point classification: the point classification process classifies each point $p_{i}$ of the point cloud $P$ into two types: existing (0) and new $(1)$. The existing points (type $=0$ ) represent surfaces in the BIM M, while the new points (type =1) belong to new elements of the building interior in comparison to the model $M$. The classification is based on a point-surface distance, which is the orthogonal distance between each point $p_{i}$ to the closest corresponding surface $\pi_{j}$ of the model $M$ (Khoshelham, 2015, 2016). The point is classified as type $=0$ if the orthogonal distance is less than a cut-off threshold $r$ and the orthogonal projection of the point on the surface falls within the surface boundary (Oude Elberink et al., 2013; Oude Elberink and Khoshelham, 2015). Otherwise, if the point-to-surface distance is larger than the cut-off threshold $r$ or its orthogonal projections do not fall within any surface boundary of the BIM, the point is labelled as type $=1$ accordingly. The threshold $\mathrm{r}$ is set according to the noise of the point cloud $\mathrm{P}$ and the error of the registration between the point cloud $\mathrm{P}$ and the BIM M. We formulate the classification of a point $p_{i}$ in the point cloud $P$ as:

$$
\text { type }\left(p_{i}\right)= \begin{cases}0 & \text { if } \exists \pi_{j}\left|\pi_{j}^{T} p_{i}\right| \leq r \text { and } \operatorname{In}\left(p_{i}, \pi_{j}\right) \\ 1 & \text { Otherwise }\end{cases}
$$

where $\left|\pi_{j}^{T} p_{i}\right|$ is the absolute orthogonal distance between the point $p_{i}$ and the surface $\pi_{j}$ of the BIM M. $\operatorname{In}($.$) denotes the$ operation on whether an orthogonal projection of the point is within the boundary of the corresponding surface.
Using the point classification method, any new elements of a building interior which are missing in the BIM will be identified in the point cloud as clusters of points of type 1. Meanwhile, the existing elements of a building which are recorded in the BIM will only contain points of type 0 . The computation of the pointto-surface distance at this step is not only useful for labelling of the points, but also provides information about location deviation of each point in the point cloud $\mathrm{P}$ and its corresponding elements in the BIM.

Surface coverage measurement: The coverage $M_{c o v}$ of a surface $\pi_{j}$ in the BIM M is measured based on the surface area that is covered by the point cloud $P$. The existing points are taken into account in the computation of the coverage of the corresponding surface. The points are first orthogonally projected on the corresponding surface in order to construct a 2D alpha-shape $\alpha$, which can be derived from the Delaunay triangulation of the projected points on the condition that the circumradius of each triangle face is smaller than an alpha radius $r_{\propto}$ (Edelsbrunner, 1992; Edelsbrunner and Mücke, 1994). The coverage $M_{c o v}$ of a surface $\pi_{j}$ is the ratio of the area of the alphashapes $\alpha$ to the area of the surface:

$$
M_{\text {cov }}\left(\pi_{j}\right)=\frac{\operatorname{area}(\alpha)}{\operatorname{area}\left(\pi_{j}\right)}
$$

where $\alpha$ denotes the alpha-shape reconstructed from the orthogonal projections of the existing points on the corresponding surface and area(.) denotes the area of a surface.

Figure 1 shows an example of coverage measurement of a surface (orange), which is partially covered by a point cloud. The alphashape $\alpha$ (green) is reconstructed with the alpha radius $r_{\alpha}<$ $0.2 m$ from the projections of the point cloud on the surface. The coverage of the surface is $M_{c o v}=0.56$, indicating that $56 \%$ of the surface area is captured in the point cloud.

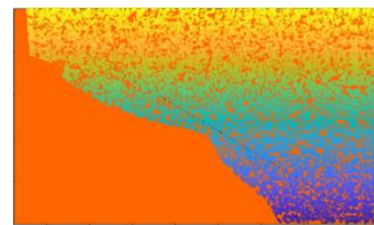

(a)

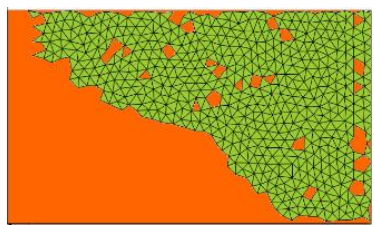

(b)
Figure 1. An example of surface coverage: (a) a surface (orange) and its corresponding point cloud; (b) a 2D alphashape (green), which is constructed from the projection of the point cloud on the surface, cover up $56 \%$ of the surface $\left(M_{\operatorname{cov}}=0.56\right)$.

The coverage $M_{\operatorname{cov}}\left(\pi_{j}\right)$ of a surface ranges from 0 to 1 . Using the surface coverage measure any redundant surface in the BIM $\mathrm{M}$, which is not present in the point cloud $P$, will be identified by a low coverage $\left(M_{\operatorname{cov}}\left(\pi_{j}\right) \approx 0\right)$ as there are no existing points (type $=0$ ) corresponding to the surface. The surfaces with higher coverage are most likely to be present in the real environment.

\section{EXPERIMENTS AND RESULTS}

\subsection{Experiments}

Experiments with both a synthetic dataset and the ISPRS benchmark data were conducted to evaluate the feasibility of the proposed method for comparison between a BIM and a point 
cloud of an indoor environment.

The synthetic dataset comprises a BIM and a point cloud of a synthetic environment. We assume that the BIM represents the previous stage of a building, which consists of a room and a corridor. Meanwhile, the point cloud captures the current stage of the building with two new walls and one removed wall in comparison with the existing BIM. The point cloud has no noise and is created by random space sampling on surfaces of the current building. Therefore, the cut-off distance can be set close to zero (here $r \approx 1 \mathrm{~mm}$ ). The synthetic dataset is shown in Figure 2.

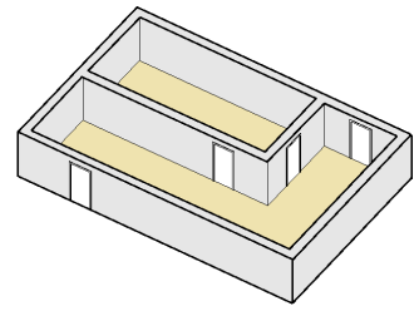

(a)

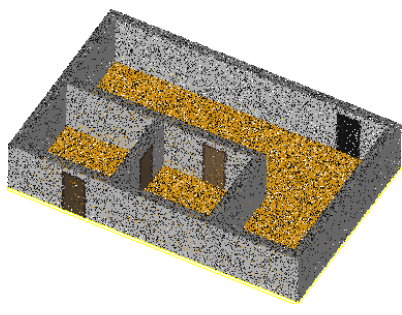

(b)
Figure 2. The synthetic dataset: (a) the BIM; (b) the point cloud.

Figure 3 shows the BIM and the corresponding point cloud of the TUB1 dataset from the ISPRS benchmark on Indoor Modelling. The point cloud was captured by a Viametris iMS3D mobile scanning system - with $3 \mathrm{~cm}$ data accuracy (Khoshelham et al., 2017). Meanwhile, the BIM, which contains visible (light grey) and interpreted surfaces (dark grey) is created from the point cloud by an expert. The visible surfaces are captured in the point cloud, while the interpreted ones are unobservable and interpreted from the visible surfaces (Khoshelham et al., 2018). In this experiment, we compare the visible building elements (i.e., walls, ceilings, and floors) in the BIM with the point cloud, which contains data points of not only the visible building elements but also open and closed doors, windows, and a low level of clutter. The cut-off threshold is set at $10 \mathrm{~cm}(\mathrm{r}=10 \mathrm{~cm})$, which corresponds to the errors of the data, the registration process of the BIM and the data, and the error in the reconstruction of the BIM. The ceilings are removed for better visualization.

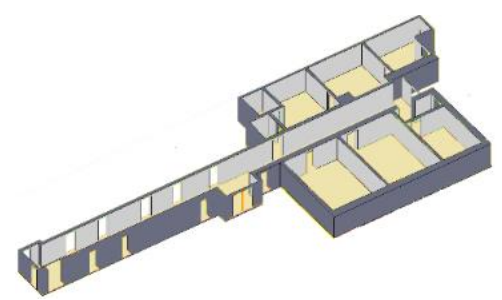

(a)

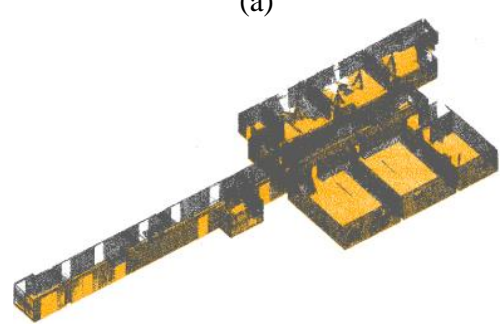

(b)

Figure 3. The ISPRS Benchmark dataset - TUB1: (a) the BIM with the visible surface marked as light grey; (b) the point cloud.

\subsection{Results}

Synthetic dataset: Figure 4 shows the result of point classification based on the point-surface distance between the point cloud and the BIM of the synthetic dataset. Changes are detected in the building in comparison with the BIM as there are clusters of points in the point cloud with large point-surface distances. As can be seen in Figure 4(a), the points belonging to the new elements of the building have a large distance from the existing BIM, up to $2.5 \mathrm{~m}$, while the remaining points of the point cloud have very small distances (close to 0 ) from the corresponding surfaces in the BIM. The points with a distance smaller than $r=1 \mathrm{~mm}$ are classified as existing points (blue) representing existing elements in the BIM as shown in Figure 4(b). Points classified as new points (yellow), which have larger point-surface distances, represent the new walls, which are not present in the existing BIM.

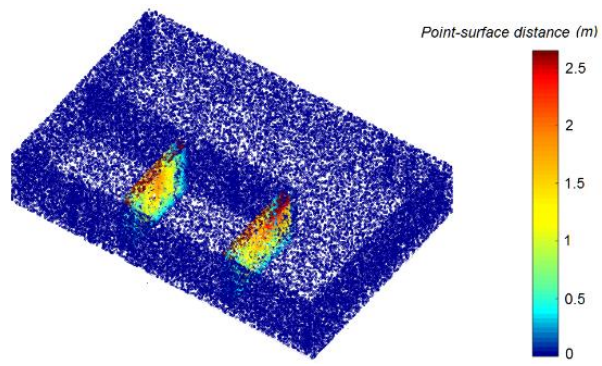

(a)

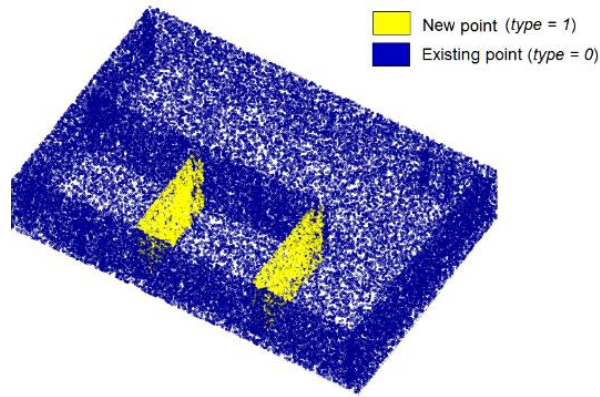

(b)

Figure 4. Comparison results of the synthetic dataset: (a) the point cloud colorized according to point-surface distances; (b) the point cloud with the result of point classification.

The coverage $M_{\text {cov }}$ of each surface of the BIM is computed based on the result of the point classification. Figure 5(a) shows the surfaces colorized according to the coverage, which ranges from $M_{c o v} \approx 0$, indicating that there is no point in the point cloud representing the surface, to $M_{c o v} \approx 1$, indicating that the surface is fully covered by points. The redundant and existing surfaces of the BIM in comparison with the point cloud are then derived from the coverage as shown in Figure 5(b). The redundant surfaces of the BIM, which do not exist in the real environment as represented by the point cloud, are identified as those surfaces that have a coverage smaller than a certain threshold set by the user (here $M_{c o v} \leq 0.3$ ), while the coverages of all correct surfaces which exist in the point cloud are larger than the threshold $\left(M_{\text {cov }}>0.3\right)$. 


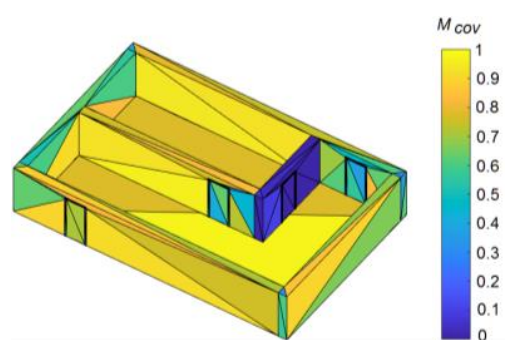

(a)

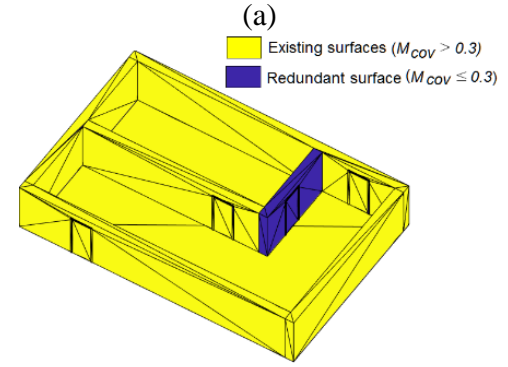

(b)

Figure 5. Comparison results of the synthetic dataset: (a) the BIM with surfaces colorized according to the coverage $M_{c o v}$; (b) the BIM with the location of redundant elements (blue) and existing surfaces (yellow).

The updating of the existing BIM to represent the current stage of the building can be guided by the locations where the changes are detected. This will facilitate efficient generation of an as-is BIM of the indoor environment.

The ISPRS benchmark dataset: The results of point classification for the comparison between the BIM and the point cloud of the TUB1 building of the ISPRS benchmark dataset are shown in Figure 6. As can be seen in Figure 6(a), the data points belonging to building structures (i.e., walls, ceilings, and floors) are close to the BIM surfaces $(\leq 10 \mathrm{~cm})$, while the points representing doors and windows, which were not reconstructed in the BIM, and the clutter (e.g., people, furniture) have larger point-surface distances. The data points are then classified based on the point-surface distance. The points are labelled as existing points $($ type $=0)$ if their point-surface distances are smaller than the cut-off threshold $(r=10 \mathrm{~cm})$. Otherwise, the points are classified as new (type $=1$ ) due to their larger point-surface distance as shown in Figure 6(b).

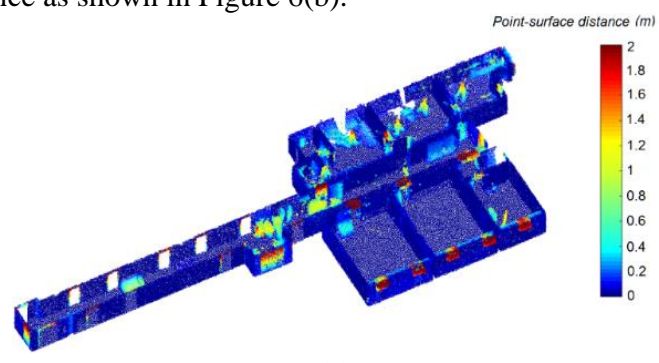

(a)

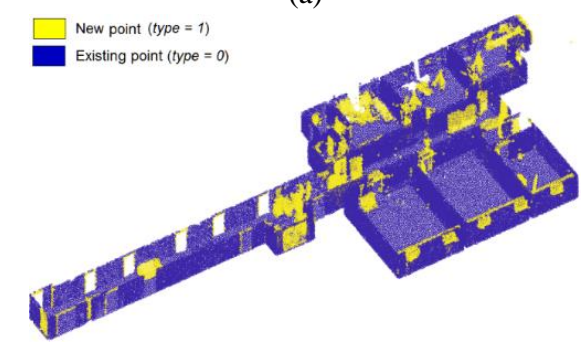

(b)

Figure 6. Comparison results of the TUB1 dataset: (a) the point cloud colorized according to point-surface distances; (b) the point cloud with the result of point classification.
Figure 7 presents the colorization of the BIM surfaces according to the coverage, and the location of redundant and existing surfaces of the BIM with respect to the point cloud. Figure 7(a) shows that several BIM surfaces are reconstructed with less supporting points than others. Users are enabled to identify the redundant surfaces in the BIM based on the surface coverage. Figure 7(b) and (c) demonstrate the ability of the proposed method in locating the redundant surfaces based on coverage thresholds of $0.2 \quad\left(M_{\operatorname{cov}}>0.2\right)$ and $0.3 \quad\left(M_{\operatorname{cov}}>0.3\right)$ respectively. The thresholds are set empirically. The surfaces which do not reach the coverage threshold are classified as redundant surfaces in the BIM. As can be seen in Figure 7(c), a surface with $M_{c o v} \approx 0.3$ is wrongly classified as redundant. This is due to the presence of furniture in the building, which leads to gaps in the point cloud.

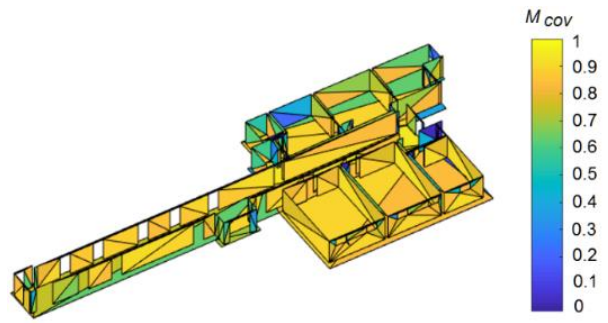

(a)

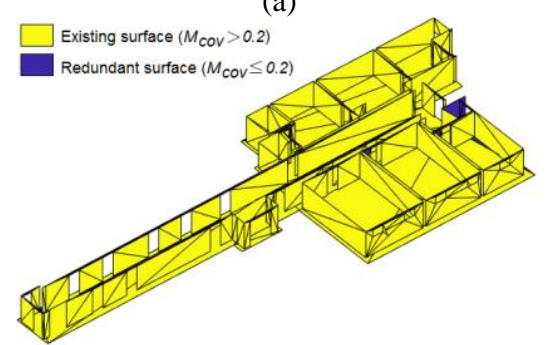

(b)

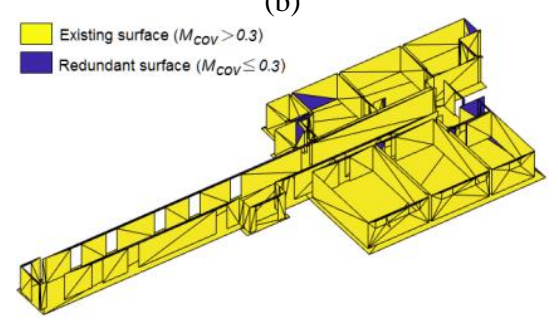

(c)

Figure 7. Comparison results of the TUB1 of the ISPRS benchmark dataset: (a) the BIM with surfaces colorized according to the coverage $M_{c o v}$; (b) and (c) the BIM with the location of redundant elements (blue) and existing surfaces (yellow) with $M_{\text {cov }} \approx 0.2$ and $M_{c o v} \approx 0.3$, respectively.

\section{CONCLUSION AND FUTURE WORK}

In this paper, an approach to building change detection through comparison between a BIM and a point cloud of an indoor environment is presented. The experiments on both a synthetic dataset and the ISPRS benchmark dataset demonstrates that the proposed approach can detect the discrepancies and locate the new elements of the building as represented by the point cloud, which are missing in the BIM, as well as redundant elements in the BIM, which do not exist in the point cloud. This enables the proposed approach to detect changes of an indoor environment and update the BIM, which is useful in a variety of applications such as temporal analyses of changes during a building's lifecycle and maintaining an as-is BIM of a building. 
The limitation of the current approach is the lack of semantic information (e.g., clutters, building elements, utilities, etc.) about the detected changes as such semantic information is not available in the point cloud of the building. Future work will focus on classification of building changes. Another direction for future research is to investigate the effect of clutter and occlusion on the change detection. We will also further evaluate the proposed method with more complex environments, which contains glass walls/windows and non-planar surface structures.

\section{ACKNOWLEDGEMENTS}

This research is supported by the Australian Research Council (DP170100153). The first author acknowledges the financial support from the University of Melbourne through Melbourne International Fee Remission and a Melbourne International Research Scholarship. The datasets used in the experiments were collected as part of the ISPRS Benchmark on Indoor Modelling.

\section{REFERENCES}

Becker, R., Falk, V., Hönen, S., Loges, S., Stumm, S., Blankenbach, J., Brell-Cokcan, S., Hildebrandt, L., Vallée, D., 2018. Bim-Towards The Entire Lifecycle. International Journal of Sustainable Development and Planning, 13(1), pp.84-95.

Choudhry, R.M., Gabriel, H.F., Khan, M.K., Azhar, S., 2017. Causes of Discrepancies between Design and Construction in Pakistan Construction Industry. Journal of Construction in Developing Countries, 22(2), pp.1-18.

CloudCompare Development Team, 2019, CloudCompare Software, version 2.9. http://www.cloudcompare.org/ (23 ${ }^{\text {rd }}$ January 2019).

Díaz-Vilariño, L., Khoshelham, K., Martínez-Sánchez, J., Arias, P., 2015. 3D modeling of building indoor spaces and closed doors from imagery and point clouds. Sensors 15, 3491-3512.

EU BIM Task Group, 2017. Handbook for the introduction of Building Information Modelling by the European Public Sector Strategic action for construction sector performance: driving value, innovation and growth. http://www.eubim.eu/wpcontent/uploads/2017/07/EUBIM_Handbook_Web_Optimized1.pdf ( $23^{\text {rd }}$ January 2019).

Edelsbrunner, H., 1992. Weighted alpha shapes. Technical Report UIUCDCS-R-92-1760, Dept. Comput. Sci., Univ. Illinois, Urbana, IL, 1992.

Edelsbrunner, H., Mücke, E.P, 1994. Three-dimensional alpha shapes. ACM Trans. Graph13(1), 43-72.

Hossain, M.A., Yeoh, J.K.W., 2018. BIM for Existing Buildings: Potential Opportunities and Barriers. In IOP Conference Series: Materials Science and Engineering, 371(1), p. 012051. IOP Publishing.

Khoshelham, K., 2015. Direct 6-DoF pose estimation from pointplane correspondences. Digital Image Computing: Techniques and Applications (DICTA), International Conference on, pp. 1-6.

Khoshelham, K., 2016. Closed-form solutions for estimating a rigid motion from plane correspondences extracted from point clouds. ISPRS Journal of Photogrammetry and Remote Sensing $114,78-91$.

Khoshelham, K., 2018. Smart Heritage: Challenges in Digitisation and Spatial Information Modelling of Historical Buildings. 2nd Workshop On Computing Techniques For SpatioTemporal Data in Archaeology And Cultural Heritage, Melbourne, Australia.

Khoshelham, K. and Díaz-Vilariño, L., 2014. 3D modelling of interior spaces: learning the language of indoor architecture. The International Archives of Photogrammetry, Remote Sensing and Spatial Information Sciences XL-5, 321-326.

Khoshelham, K., Díaz Vilariño, L., Peter, M., Kang, Z., Acharya, D., 2017. The ISPRS Benchmark on Indoor Modelling. The International Archives of Photogrammetry, Remote Sensing and Spatial Information Sciences XLII-2/W7, 367-372.

Khoshelham, K., Tran, H., Díaz-Vilariño, L., Peter, M., Kang, Z., Acharya, D., 2018. An Evaluation Framework For Benchmarking Indoor Modelling Methods. ISPRS-International Archives of the Photogrammetry, Remote Sensing and Spatial Information Sciences, 624, pp.297-302.

Mura, C., Mattausch, O., Pajarola, R., 2016. Piecewise-planar Reconstruction of Multi-room Interiors with Arbitrary Wall Arrangements. Computer Graphics Forum 35(7), 179-188.

Nikoohemat, S., Koeva, M., Oude Elberink, S.J., Lemmen, C.H.J., 2018. Change Detection from Point Clouds to Support Indoor $3 \mathrm{~d}$ Cadastre. International Archives of the Photogrammetry, Remote Sensing \& Spatial Information Sciences, 42(4).

Oude Elberink, S., Khoshelham, K., 2015. Automatic extraction of railroad centerlines from mobile laser scanning data. Remote Sensing 7, 5565-5583.

Oude Elberink, S., Khoshelham, K., Arastounia, M., Diaz Benito, D., 2013. Rail track detection and modelling in mobile laser scanner data. ISPRS Annals of the Photogrammetry, Remote Sensing and Spatial Information Sciences. II-5/W2, 223-228.

Previtali, M., Díaz-Vilariño, L., Scaioni, M., 2018. Indoor building reconstruction from occluded point clouds using graphcut and ray-tracing. Applied Sciences, 8(9), p.1529.

Tran, H., Khoshelham, K., Kealy, A., Díaz Vilariño, L., 2017. Extracting topological relations between indoor spaces from point clouds. ISPRS Annals of the Photogrammetry, Remote Sensing and Spatial Information Sciences 4, 401- 406.

Tran, H., Khoshelham, K., Kealy, A., Díaz-Vilariño, L., 2018. Shape Grammar Approach to 3D Modelling of Indoor Environments Using Point Clouds. Journal of Computing in Civil Engineering, 33(1), p.04018055.

Tran, H., Khoshelham, K., Kealy, A., 2019. Geometric comparison and quality evaluation of 3D models of indoor environments, ISPRS Journal of Photogrammetry and Remote Sensing 149, 29-39.

Wang, J., Sun, W., Shou, W., Wang, X., Wu, C., Chong, H.Y., Liu, Y., Sun, C., 2015. Integrating BIM and LiDAR for real-time construction quality control. Journal of Intelligent \& Robotic Systems, 79(3-4), pp.417-432. 